\title{
O papel das emoções na inclusão laboral de pessoas com deficiência.
}

\author{
Marta Nogueira ${ }^{1}$ e Célia Sousa ${ }^{2}$ \\ ${ }^{1}$ Doctoral student - Universidad de Extremadura, Espanha; ESECS-IPLeiria, Portugal | \\ marta.nogueira@ipleiria.pt | https://orcid.org/0000-0002-9503-4451 \\ 2 ESECS-IPLeiria,CRID,CICS.NOVA.IPLeiria,Portugal | celia.sousa@ipleiria.pt | \\ https://orcid.org/0000-0002-5113-9323
}

\begin{abstract}
Resumo: A inclusão laboral de pessoas com deficiência (PcD's) é um dos pontos mais importantes para a sua inclusão na sociedade. Apesar da constante evolução na legislação, as pessoas com deficiência ainda não têm o mesmo acesso às oportunidades de trabalho que os seus colegas sem deficiência. É premente combater o estigma relacionado a esta população. Nesse sentido, este estudo contribuiu para o aprofundamento do conhecimento que as pessoas com deficiência (PcD's) têm sobre as suas emoções no local de trabalho, bem como do seu processo de inclusão, trazendo sugestões de melhoria sobre esta questão social tão pertinente. Foram definidos os seguintes objetivos específicos: conhecer o grau de consciência que os trabalhadores com deficiência têm das suas emoções nas interações diárias no seu local de trabalho e, verificar em que medida a Convenção sobre os Direitos das Pessoas com Deficiência melhorou o processo de inclusão. Neste estudo, optou-se pela pesquisa qualitativa, com recurso à entrevista semiestruturada. $\mathrm{A}$ amostra foi constituída por 50 trabalhadores com deficiência, funcionários do Grupo Auchan Portugal. Os resultados mostraram que as PcD's reconhecem as suas emoções quando interagem com os seus colegas de trabalho. No entanto, a maioria destas pessoas disfarça o desconforto sentido, não comunicando de forma clara que emoções sentiu. Além disso, verificou-se que, apesar da maioria das PcD's se sentir incluída, sugere uma maior sensibilidade da entidade empregadora, trabalho adaptado à pessoa e à sua deficiência $e$ mais acessibilidades no local de trabalho.
\end{abstract}

Palavras-chave: Deficiência; Emoções; Inclusão; Trabalho.

The Role of Emotions in the Work Inclusion of People with Disabilities.

\begin{abstract}
The labor inclusion of people with disabilities (PcD's) is one of the most important points for their inclusion in society. Despite the constant evolution in legislation, people with disabilities still don't have the same access to job opportunities as their non-disabled colleagues. It is urgent to fight the stigma related to this population. In this sense, this study contributed to the deepening of the knowledge that people with disabilities (PcD's) have about their emotions in the workplace, as well as about their inclusion process, bringing suggestions for improvement about this very pertinent social issue. The following specific objectives were defined: to know the degree of awareness that workers with disabilities have about their emotions in their daily interactions at their workplace and, to verify to what extent the Convention on the Rights of Persons with Disabilities has improved the inclusion process. In this study, qualitative research was chosen, using semi-structured interviews. The sample was composed of 50 workers with disabilities, employees of the Auchan Portugal Group. The results showed that PwD's recognize their emotions when interacting with their co-workers. However, most of these people disguise the discomfort felt, not communicating clearly which emotions they felt. In addition, it was found that, although most PcD's feel included, it suggests greater sensitivity from the employer, work adapted to the person and their disability, and more accessibility in the workplace.
\end{abstract}

Keywords: Disability; Emotions; Inclusion; Work.

\section{Introdução}

Devido à crescente ênfase global nas políticas de bem-estar para o trabalho, um número crescente de pessoas com deficiência $(\mathrm{PcD})$ ingressou na força de trabalho. No entanto, a maioria dos estudos encontrados sobre o tema focaram-se essencialmente nas práticas da empresa para acomodar as $\mathrm{PcD}$, com uma compreensão limitada sobre os fatores psicológicos e emocionais que afetam a sua inclusão (Xiji et al., 2019). 
As $\mathrm{PcD}$ enfrentam constantemente várias barreiras quando se trata de emprego Estas barreiras começam pela procura de emprego, sendo que muitas destas pessoas consideram esta fase complexa e geradora de um elevado nível de stress (Rueda \& Andrade, 2016); no recrutamento e selecção, em que a contratação destas pessoas é motivada apenas pela natureza obrigatória da lei e pela baixa oferta de vagas (Lorenzo \& Silva, 2017); a falta de formação por parte das empresas para trabalharem com $\mathrm{PcD}$ (Carreras \& Moreno, 2011; Felizardo et al., 2016); e a falta de acompanhamento contínuo das PcD após a sua contratação (Rosa et al.,2019).

Além disso, o sucesso profissional das PcD, assim como de todas as pessoas em geral, depende em grande parte da gestão das emoções (Arandiga, 2009). De acordo com Alkozei et al. (2018), as emoções e sentimentos contêm informações importantes que ajudam os gerentes e os funcionários a terem um melhor desempenho no local de trabalho. No local de trabalho, se os indivíduos desejam atingir as suas metas e objetivos, é imperativo que eles formem relações de trabalho apropriadas com outras pessoas (Naderi, 2012; Yang et al., 2018). A Inteligência Emocional altamente desenvolvida, ajuda os indivíduos a identificar os sentimentos e traços comportamentais de outros (Naseem, 2018; Thory, 2016).

Em relação aos trabalhadores com deficiência, foi verificado que estes muitas vezes não têm a oportunidade de expressar as suas emoções num contexto de trabalho e, quando o fazem, acabam por ser ignorados (Coutinho et al., 2017). Muitas destas emoções relacionam-se com sentimentos negativos vividos pelas PcD's durante o seu dia a dia de trabalho, devido a atitudes de preconceito e estigma por parte colegas e gestores. Tais atitudes acabam por gerar nestas pessoas um aumento dos níveis de ansiedade, stress, dificuldades em permanecer no emprego (Shahin et al., 2020), para além de despedimentos ilegais e incapacidade de demonstrar e pôr em prática as suas competências (Ripat \& Woodgate, 2017; Lieketseng \& Lorenzo, 2016). No estudo de Pinheiro e Dellatorre (2015) verificou-se que os colegas e chefes tinham sentimentos de pena, fragilidade e inferioridade para com as PcD's. Verificou-se também neste estudo que as PcD's evitavam pedir qualquer tipo de ajuda aos colegas ou superiores, principalmente devido a sentimentos de vergonha. Além disso, muitos empregadores e colegas de trabalho das PCD's, não sabem como lidar com as emoções destes trabalhadores (Zappella, 2015). As razões pelas quais as PcD's omitem o que sentem no local de trabalho, relacionam-se com o preconceito, a ignorância sobre a diferença, e a falta de cumprimento da legislação, o que faz com que se sintam desconfortáveis (Santos, 2014). No estudo de Sherbin e Kennedy (2017), foi verificado que os funcionários com deficiência que apresentaram as suas ideias, emoções e pontos de vista à organização foram totalmente ignorados.

Nesse seguimento, foram definidos os seguintes objetivos: conhecer o grau de consciência que os trabalhadores com deficiência têm das suas emoções nas interações diárias no seu local de trabalho e, verificar em que medida a Convenção sobre os Direitos das Pessoas com Deficiência melhorou o processo de inclusão. O objetivo deste estudo é contribuir para o reconhecimento unânime do direito ao trabalho, uma vez que o emprego é de importância fulcral no processo de inclusão social e emancipação económica das PcD (Gonçalves \& Nogueira, 2012).

\section{Metodologia}

No presente estudo optou-se pela metodologia qualitativa, com recurso à entrevista semiestruturada.

\subsection{Amostra}

A amostra foi constituída por 50 PcD, empregados dos hipermercados do Grupo Auchan de Norte a Sul de Portugal, na sua maioria homens $(60 \%)$ e com idades compreendidas entre os 30 e os 49 anos. 
Em relação ao tipo de deficiência, cerca de metade dos participantes tinha Deficiência Intelectual (50\%); seguidos pelos participantes com Deficiência Física ou Motora (32\%); com Deficiência Visual (10\%) e Deficiência Auditiva (8\%). O Auchan Portugal, representa uma rede de hipermercados e é considerado um exemplo de boas práticas na área do recrutamento e integração de $\mathrm{PcD}$.

\subsection{Recolha dos Dados}

A recolha de ocorreu de forma presencial, entre fevereiro de 2018 e março de 2020 , com deslocação às várias lojas do país, mediante agendamento prévio com o responsável de mercado e, com recurso a registo digital, com o consentimento informado de cada trabalhador

Neste estudo, foi escolhida a metodologia qualitativa, com a utilização de entrevistas semiestruturadas com os trabalhadores com deficiência. O guião da entrevista aplicado aos participantes foi construído de raiz tendo em conta os objetivos estipulados e consistia em cinco blocos de perguntas; alguns destes blocos continham subparágrafos (blocos 2, 3, e 5). A parte inicial da entrevista continha a caracterização sociodemográfica do entrevistado (nome, idade, sexo, qualificação académica, tipo de deficiência, e número de meses/anos de experiência de trabalho). Para avaliação da Inteligência Emocional, o guião de entrevista continha duas perguntas, sendo que uma divide-se em sub perguntas de resposta aberta. Alguns exemplos de uma dessas questões são: "Existe alguma situação que habitualmente origina uma emoção mais intensa? Qual situação? O que sente?”.

\subsection{Análise dos Dados}

O tratamento dos dados recolhidos incluiu a análise de conteúdo, com base em categorias de análise pré-definidas resultantes da revisão bibliográfica e dos objetivos de investigação; e a codificação das respostas. Foi utilizado um software de processamento de dados qualitativo: NVivo 10.1.10, um programa de software usado para investigações qualitativas e de métodos mistos (Bringer et al., 2004). Especificamente, é utilizado para a análise de texto não estruturado, áudio, vídeo e dados de imagem, incluindo (mas não se limitando a) entrevistas, pesquisas e artigos de jornal (Johnston, 2006). Este software permite recolher e analisar o conteúdo das transcrições, mantendo uma visão global e ordenada dos códigos assim obtidos.

\section{Resultados}

Os resultados da entrevista às $\mathrm{PCD}$, revelaram que o sentimento/emoção que mais caracteriza a vida profissional diária destas pessoas foi o Orgulho, como podemos verificar através de alguns excertos: "Orgulho, em mim e no trabalho que faço, cumpro as regras do dia" (Operador de supermercado); "Orgulho no que faço, gosto do que faço e eles dizem-me que sei trabalhar bem, que sou simpático para os clientes" (Operador de supermercado).

Uma situação que normalmente gera uma emoção mais intensa no trabalho foi "Ser chamado à atenção no meu trabalho", como podemos ver através de alguns exemplos relatados pelos participantes "Eles vêm ao meu trabalho para chamar a minha atenção para certas coisas e, ao mesmo tempo, dão-me ordens. Isso entristece-me. Ninguém gosta de ser chamado à atenção, certo?" (Operador de supermercado); "Quando o trabalho não corre como eu gostaria e sou chamado pelos meus colegas, e muitas vezes não é da melhor maneira" (Operador de hipermercado). 
Outras situações foram: "Indiferença, discriminação e exclusão", causando um sentimento de tristeza e frustração: "Os clientes não querem ser atendidos por mim porque eu tenho uma deficiência. Sinto frequentemente esta indiferença" (Assistente de Operador de supermercado); "Sinto indiferença por parte dos colegas de trabalho. Não me sinto compreendido. Não é só integrar, mas também compreender e defender" (Telefonista); "Por vezes sinto que sou excluído das reuniões de trabalho. Isso deixa-me um pouco insatisfeito" (Telefonista).

A maioria das PCD's não diz claramente à outra qual a emoção que sentiu e disfarça o desconforto das emoções que sentiu; dizem ao outro qual o comportamento que lhes causou desconforto.

Os resultados das entrevistas mostraram também que proporcionar uma maior inserção profissional da PcD, gostariam de ver modificado "Mais oportunidades de emprego", uma "Maior sensibilidade por parte das entidades empregadoras", e o "Trabalho adaptado à pessoa e à sua deficiência".

\section{Discussão dos resultados}

Os resultados da entrevista realizada à $P c D$ indicaram que a emoção que mais caracteriza o dia-a-dia no trabalho da PcD é o Orgulho, nomeadamente o orgulho em ter um emprego, em ser autónomo e ter a possibilidade de contribuir para a sociedade. Também no estudo de Souza (2019) que teve como objetivo avaliar as emoções de pessoas com deficiência intelectual durante 0 trabalho, 0 sentimento mais frequentemente reportado foi o orgulho, relacionado à dignidade de terem um emprego.

As situações que habitualmente geram uma emoção intensa em contexto laboral foram "Chamarem-no atenção pelo seu trabalho"; a "Indiferença, discriminação e exclusão", originando um sentimento de tristeza e frustração. Resultados semelhantes foram encontrados por Lindsay et al. (2017) que verificaram que uma reação negativa por parte dos colegas de trabalho de $\operatorname{PcD}$ (discriminação) levou a que estes tivessem experiências e sentimentos negativos, como o aumento dos níveis de ansiedade, stress e dificuldades em permanecer no emprego.

Os resultados mostraram que a maioria dos $\mathrm{PCD}$ não diz claramente ao outro qual a emoção sentida e disfarça o desconforto das emoções sentidas. Estes resultados vão de encontro aos de Coutinho et al. (2017) que verificaram que os funcionários com deficiência muitas vezes não têm oportunidade de expressar suas opiniões e emoções. Estas pessoas acabam por ser discriminadas pelos empregadores, assim como pelos colegas, por causa de conceitos erróneos sobre as suas capacidades, reforçando uma visão preconceituosa acerca delas e impedindo que expressem abertamente as suas opiniões e emoções (Coutinho et al., 2017). Também Sherbin e Kennedy (2017) concluíram que grande parte dos trabalhadores com deficiência não tem a possibilidade de expor as suas ideias, emoções, e sentimentos no ambiente de trabalho e, aqueles que o fazem, acabam por ser ignorados pelas entidades empregadoras.

Por fim, os resultados mostraram que a fim de proporcionar uma maior inserção profissional da PcD, gostariam de ver modificado "Mais oportunidades de emprego", uma "Maior sensibilidade por parte das entidades empregadoras", e o "Trabalho adaptado à pessoa e à sua deficiência".

\section{Conclusões}

A presente investigação veio dar a conhecer um pouco da realidade vivida pelas $P c D$ no local de trabalho, nomeadamente no que concerne às suas emoções. Um melhor entendimento acerca das emoções destas pessoas durante a sua jornada de trabalho, abre caminhos para que sejam feitas melhorias de modo a aumentar a sua qualidade de vida laboral. 
É muito comum as $\mathrm{PcD}$ desenvolverem certos problemas tais como baixa autoestima, baixa autoconfiança, aumento nos níveis stress e ansiedade, etc, devido a várias barreiras que enfrentam no processo de inclusão laboral. A inteligência emocional pode ser um importante aliado nesse processo, pois saber lidar com as próprias emoções é essencial para superar os desafios do dia a dia. Assim, ao conhecermos melhor os sentimentos e emoções das PcD, podemos entender o que estas pessoas enfrentam diariamente e, assim, ajudá-las, o que pode conduzir a uma melhor inclusão e, consequentemente, a uma maior permanência no trabalho e a uma maior produtividade. Infelizmente, em muitas empresas ainda há uma cultura discriminatória, em que as entidades evitam contratar PcD, uma vez que têm uma conceção errónea acerca das mesmas, julgam-nas como pouco produtivas, pouco autónomas e incapazes de realizar as tarefas adequadamente. Frequentemente, estas pessoas são alvo de discriminação direta, não possuem apoio adequado e os colegas/superiores nem sempre são formados adequadamente para lidar com elas, o que se traduz numa difícil integração, tendo consequências diretas na sua produtividade. No entanto, tal pode acontecer com uma pessoa sem deficiência, caso não tenha formação e suporte adequado. Portanto, é pertinente colocarmo-nos no lugar das $\mathrm{PcD}$, compreender as suas emoções, as suas vivências diárias no local de trabalho, de forma a contribuir para que tenham uma boa qualidade de vida e possam desempenhar corretamente as suas funções.

Os resultados aqui apresentados e as conclusões obtidas não podem ser generalizados, uma vez que estes dizem respeito a um contexto expecífico e não à sociedade em geral, mas poderão ser pontos orientadores de trabalhos que futuramente possam ser realizados sobre o papel das emoções na inclusão laboral de PcD. Contudo, futuramente devem ser definidas por parte dos profissionais, estratégias individuais para cada PcD, como por exemplo, a gestão das emoções no local de trabalho, a formação adequada às necessidades de cada um, de forma a poder ser incluída no local de trabalho mais facilmente e sem qualquer sofrimento psicológico. Seria importante também aprofundar mais questões, como as vivências pessoais, o desempenho e a motivação das PcD, que emergem dos dados recolhidos como interessantes pistas para a caracterização e compreensão desta realidade social.

A principal limitação deste estudo está relacionada com o fator temporal, na medida em que foi imposto um prazo limite para a elaboração do mesmo. Outra limitação relacionase com o tamanho reduzido da amostra, portanto seria importante futuramente realizar um estudo com uma amostra maior.

\section{Referências}

Alkozei, A., Smith, R., Demers, L., \& Weber, M. (2018). Increases in Emotional Intelligence After an Online Training Program Are Associated With Better Decision-Making on the lowa Gambling Task. Psychological Reports, 122(1). https://doi.org/10.1177/0033294118771705

Arándiga A. (2009). La inteligência emocional de los padres y de los hijos. Ediciones Pirámide.

Bringer, J., Johnston, L., \& Brackenridge, C.(2004). Maximising transparency in a doctoral thesis: The complexities of writing about the use of QSR*NVIVO within a grounded theory study. Qualitative Research, 4, 247-265. https://doi.org/10.1177/1468794104044434.

Carreras, S., \& Moreno, W. (2011). Perspectivas de la experiencia gerencial hacia empleados con discapacidad intelectual. Cuaderno de Investigación, 26, 183-199.

Coutinho, B., França, I., Coura, A., Medeiros, K., \& Aragão, J. (2017).Qualidade de vida no trabalho de pessoas com deficiência física. Trabalho, Educação e Saúde, 15(2), 561573.https://doi.org/10.1590/1981-7746-sol00061

Felizardo, P., Stela Ronchi, F., Robaina, G., \& Paiva, E. (2016). Inclusão de pessoas com deficiência nas organizações e impacto no clima organizacional (CO). Revista FAE, 1, 159176.

Gonçalves, J. (coord) \&amp; Nogueira, J. (2012). O Emprego das Pessoas com Deficiências ou Incapacidade - Uma abordagem pela igualdade de oportunidades. MSSS - Gabinete de estratégia e planeamento.http://www.cartasocial.pt/pdf/emprego_pdi.pdf. 
Johnston, L. H. (2006). Software and method: Reflections on teaching and using QSRNVivo in doctoral research. International Journal of Social Research Methodology, 9, 379-391.

Lieketseng, N. \& Lorenzo, T. (2016). Enhancing the Public Sector's Capacity for Inclusive Economic Participation of Disabled Youth in Rural Communities. African Journal of Disability, 5(1), 189. https://doi.org/10.4102/ajod.v5i1.189.

Lindsay, S., McPherson, A., \& Maxwell, J. (2017). Perspectives of School-Work Transitions among Youth with Spina Bifida, Their Parents and Health Care Providers. Disability and Rehabilitation, 39, 641-652. https://doi.org/10.3109/09638288.2016.1153161

Lorenzo, S., \& Silva, N. (2020). Dificuldades para contratação de pessoas com deficiência nas empresas. Revista Laborativa, 9(1), 46-69.

Naderi, A. N. (2012). Teachers: emotional intelligence, job satisfaction, and organizationa commitment. Journal of workplace Learning, 24(4), 256-269.

Naseem, K. (2018). Job Stress, happiness and life satisfaction: the moderating role of emotional intelligence empirical study in telecommunication sector pakistan. Journal of Social Sciences and Humanity Studies, 4(1), 7-14.

Pinheiro, L. \& Dellatorre, R. (2015). Desafios da Inclusão de Pessoas com Deficiência no Mercado de Trabalho: Um Estudo sobre a Percepção dos Envolvidos. Perspectiva, Erechim, 39(148), 95-109. https://www.uricer.edu.br/site/pdfs/perspectiva/148_537.p. 